\title{
Models of Cuspy Triaxial Galaxies
}

\section{Citation}

Holley\#Bockelmann, Kelly, J. Christopher Mihos, Steinn Sigurdsson, and Lars Hernquist. 2001. "Models of Cuspy Triaxial Galaxies." The Astrophysical Journal 549 (2): 862-70. https:// doi.org/10.1086/319453.

\section{Permanent link}

http://nrs.harvard.edu/urn-3:HUL.InstRepos:41381853

\section{Terms of Use}

This article was downloaded from Harvard University's DASH repository, and is made available under the terms and conditions applicable to Other Posted Material, as set forth at http:// nrs.harvard.edu/urn-3:HUL.InstRepos:dash.current.terms-of-use\#LAA

\section{Share Your Story}

The Harvard community has made this article openly available.

Please share how this access benefits you. Submit a story.

\section{Accessibility}




\title{
MODELS OF CUSPY TRIAXIAL GALAXIES
}

\author{
Kelly Holley-BockelmanN, ${ }^{1}$ J. Christopher Minos, ${ }^{1}$ Steinn Sigurdsson, ${ }^{2}$ and Lars Hernquist ${ }^{3}$ \\ Received 2000 July 18; accepted 2000 November 14
}

\begin{abstract}
We construct numerical models of mildly triaxial elliptical galaxies with central density cusps. Using a technique we call "adiabatic squeezing," we begin with a spherical $\gamma=1$ Hernquist model and apply a drag to the velocities of the particles along each principal axis. The final models are stable in isolation, preserving their density structure and figure shape over many dynamical timescales. The density profile and axial ratios compare well to the observed properties of elliptical galaxies. The orbital structure of these models show a mixture of tubes, boxes, and boxlets, as expected for triaxial systems, with very few chaotic orbits. These $N$-body realizations of cuspy triaxial galaxies provide a basis for the study of dynamical evolution of elliptical galaxies.
\end{abstract}

Subject headings: galaxies: elliptical and lenticular, cD - galaxies: kinematics and dynamics galaxies: structure - methods: $n$-body simulations

\section{INTRODUCTION}

There is good reason to believe that many elliptical galaxies are at least moderately triaxial systems. Observationally, the distribution of isophotal shapes of elliptical galaxies argues that there are few perfectly spherical or axisymmetric members of the present-day elliptical population (Tremblay \& Merritt 1995; Ryden 1996). Using morphological and kinematic data, Franx, Illingworth, \& de Zeeuw (1991) have shown that elliptical galaxies show mild triaxiality, while more recently Bak \& Statler (2000) have arrived at a similar conclusion by modeling the kinematics of ellipticals. There is strong theoretical support for triaxiality as well. Under the gravitational collapse model of elliptical galaxy formation, models of protogalaxies undergo a nonspherical collapse, which results in a virialized system that is decidedly triaxial (Dubinski \& Carlberg 1991). Similarly, in the hierarchical merging picture, the remnants of simulated galaxy mergers show strong triaxiality (e.g., Hernquist 1992; Barnes 1988, 1992; Hernquist 1993), even when multiple mergers are considered (Weil \& Hernquist 1996). Either model of elliptical galaxy formation predicts that a dynamically young elliptical galaxy should possess a fair degree of triaxiality, although subsequent evolution may drive the system toward axisymmetry.

There is also good reason to believe that many ellipticals have cuspy density profiles. While elliptical galaxies were once characterized by flat, isothermal cores, studies using the Hubble Space Telescope have shown that, in fact, the density profile of ellipticals rarely becomes perfectly flat in the inner region. Instead, the density profile continues to rise as $\rho \propto \boldsymbol{r}^{-\gamma}$, where $0.25<\gamma<2$ (e.g., Lauer et al. 1995; Byun et al. 1996; Gebhardt et al. 1996; Faber et al. 1997). Furthermore, correlations exist between the cusp slope $\gamma$ and the global properties of the galaxy, including shape in the form of boxy or disky isophotes (Faber et al. 1997).

\footnotetext{
${ }^{1}$ Department of Astronomy, Case Western Reserve University, 10900 Euclid Avenue, Cleveland, OH 44106; kelly@eor.astr.cwru.edu, hos@burro.astr.cwru.edu.

${ }^{2}$ Department of Astronomy and Astrophysics, Pennsylvania State University, 525 Davey Laboratory, University Park, PA 16802; steinn@astro.psu.edu.

${ }^{3}$ Department of Astronomy, Harvard University, 60 Garden Street, Cambridge, MA 02138; lhernqui@cfa.harvard.edu.
}

Dynamical arguments suggest also that the presence of a strong central cusp $(\gamma>1)$ acts to drive chaos in the orbit families that populate the galaxy, driving the system away from strong triaxiality (e.g., Gerhard \& Binney 1985; Norman, May, \& van Albada 1985; Merritt \& Valluri 1996; Holley-Bockelmann et al. 2001).

While real elliptical galaxies most probably are cuspy and moderately triaxial, most self-consistent modeling of elliptical galaxies to date has focused either on cuspy spherical or axisymmetric models or triaxial models with constant density cores (i.e., $\gamma=0$ ). The perfect ellipsoid of de Zeeuw (1985) allows for differing degrees of triaxiality but has a flat $\gamma=0$ core. Self-consistent $N$-body models of elliptical galaxies used in studies of black hole growth have either examined triaxiality in a $\gamma \sim 0$ profile (e.g., Norman et al. 1985; Merritt \& Quinlan 1998) or focused on cuspy models that are spherical (Sigurdsson, Hernquist, \& Quinlan 1995; Quinlan \& Hernquist 1997) or axisymmetric (van der Marel, Sigurdsson, \& Hernquist 1997). The problem lies in the lack of an analytic distribution function for systems that are both cuspy and triaxial; without such a distribution function, generating initial conditions for simulations is difficult.

Here we employ an alternative approach to generating triaxial galaxy models with well-defined shapes and cusps. We apply an adiabatic drag on the particles in a spherical $\gamma=1$ Hernquist model to mold the system into triaxial shape. This process preserves the cusp slope and results in models that are moderately triaxial and have axis ratios that are reasonably constant with radius. More importantly, the models are stable, exhibiting little evolution in their structural properties over many crossing times. We characterize the orbit families that populate such models and find a rich mixture of tubes, boxes, and boxlets, with very few chaotic orbits. This technique has also been applied successfully in a $\gamma=0$ model and can, in principle, generate models with a wide range in cusp slopes. These well-defined models thus present a useful tool for studying the evolution of elliptical galaxies or triaxial halos.

\section{MODELING TECHNIQUE}

Because there is no known distribution function for triaxial elliptical galaxies, we cannot simply sample an analytic distribution function to generate initial conditions for 
the model. Instead, we begin with an object with a known distribution function - a spherical, isotropic model that has a distribution function $f(E)$ that is a function only of energy - and mold that model into a triaxial shape by applying an artificial drag on the particles.

We begin with a spherical Hernquist (1990) model, which has the density profile

$$
\rho(r)=\frac{M}{2 \pi} \frac{a}{r} \frac{1}{(r+a)^{3}},
$$

where $M$ is the total mass and $a$ is a scale length. In projection, this model follows closely an $R^{1 / 4}$ law and possesses a three-dimensional central density cusp of the form $\rho \sim r^{-1}$ as $r \rightarrow 0$. This model belongs to a family of $\gamma$ models (Dehnen 1993; Tremaine et al. 1994) whose density profile is given by

$$
\rho_{\gamma}(r)=\frac{(3-\gamma) M}{4 \pi a^{3}}\left(\frac{r}{a}\right)^{-\gamma}\left(1+\frac{r}{a}\right)^{-(4-\gamma)},
$$

such that the Hernquist profile corresponds to $\gamma=1$. The Hernquist model has the distribution function (Hernquist 1990)

$$
\begin{aligned}
f(E)= & \frac{M}{8 \sqrt{2} \pi^{3} a^{3} v_{g}^{3}} \frac{1}{\left(1-q^{2}\right)^{5 / 2}}\left[3 \sin ^{-1} q\right. \\
& \left.+q\left(1-q^{2}\right)^{1 / 2}\left(1-2 q^{2}\right)\left(8 q^{4}-8 q^{2}-3\right)\right],
\end{aligned}
$$

where

$$
q=\sqrt{-\frac{a}{G M} E}
$$

and

$$
v_{g}=\left(\frac{G M}{a}\right)^{1 / 2} .
$$

To generate the initial spherical model, we sample this distribution using a multimass scheme developed by Sigurdsson et al. (1995), wherein particles have a mass that is roughly inversely proportional to their pericentric radius. In this manner, in the central regions of the model the particle number density rises faster than the mass density, permitting better resolution and sampling of the phase space distribution of the nuclear region. Because of the selfconsistent field (SCF) method of force calculation (see below), this multimass technique does not introduce any spurious mass segregation or relaxation into the model.

Once the initial model is constructed, we apply a timevarying adiabatic drag on the motions of the particles to mold the system into a triaxial shape. The construction is a three-step process. A drag is first applied to the $z$-axis of the model while forcing axisymmetry on the system through the zeroing of odd terms in the SCF expansion of the gravitational potential. This drag term is smoothly turned on as

$$
\xi(t)=\xi_{0}\left[3\left(\frac{t}{t_{\text {grow }}}\right)^{2}-2\left(\frac{t}{t_{\text {grow }}}\right)^{3}\right],
$$

where $\xi$ is the drag factor applied to the velocities and $t_{\text {grow }}$ is the timescale over which the drag grows. This form results in a smooth development of the drag factor, as $\dot{\xi}=0$ at $t=0$ and $t=t_{\text {grow }}$. The drag is then used to modify particle velocities as

$$
v^{\prime}=v \frac{1-0.5 \xi \Delta t}{1+0.5 \xi \Delta t},
$$

which ensures a smooth onset and termination of the drag coefficients. After $t=t_{\text {grow }}$, the drag remains at full strength for a time $t_{\text {drag }}$, after which it is slowly turned off over a time $t_{\text {decay }}$. For the models discussed here, we employed $t_{\text {grow }}=$ $t_{\text {decay }}=10$ and $t_{\text {drag }}=30$, compared to a half-mass dynamical timescale for the model of $t_{\mathrm{dyn}, 1 / 2}=8.33$.

During the squeezing process, the model contracts in radius as the system adjusts to the induced change in binding energy. Once the $z$-dragging is complete, the radius and velocity vectors of the particles are rescaled so that the system is in equilibrium with scale radius $a=1$. The axisymmetry requirement is then relaxed, and dragging ensues along the $y$-axis on similar timescales but with a different drag coefficient $\left(\xi_{0}\right)$. When $y$-dragging is complete, the system is again rescaled to $a=1$ and is evolved forward in time with all dragging shut off, allowing it to settle into an equilibrium configuration.

The models are evolved using the self-consistent field method of Hernquist \& Ostriker (1992). The SCF method expresses the density and potential as an expansion in a set of basis functions, the lowest order of which represents the spherical Hernquist density profile. The expansion coefficients are determined from the particle distribution, using $n$ radial terms and $l, m$ angular terms. For the high-resolution $N=512,000$ particle models shown in the next section, we use $n_{\max }=10, m_{\max }=l_{\max }=6$, while for smaller models $(N=128,000)$, we use $n_{\max }=6, m_{\max }=l_{\max }=4$ to reduce root- $N$ noise in the determination of the coefficients. The orbits of the particles are integrated using a high-order hermite integrator with variable time steps.

\section{GENERATING A TRIAXIAL MODEL}

We demonstrate this process with an $N=512,000$ particle $M=a=1$ Hernquist model. For this model, we use squeezing coefficients $\xi_{0, z}=0.03$ and $\xi_{0, y}=0.025$, with $t_{\text {grow }}=t_{\text {decay }}=10$ and $t_{\text {drag }}=30$.

During the squeezing, the system retains its initial density profile (once the model is rescaled so that $a=1$ ). Figure 1 shows the density profile of the model throughout the squeezing process. The profile is virtually unchanged from its original shape, save for a minor "kink" in the density profile at large radius $(r \sim 50)$. Certainly, out at this distance the dynamical timescale is significantly longer than the dragging timescale, and these particles simply have not responded as strongly to squeezing. The density is extremely low at this distance, however; only $4 \%$ of the total mass is found outside this radius. The bulk of the system is well within this radius, where the density profile is very well behaved. To amplify this, we show in Figure 2 the slope of the central density cusp as a function of time. Again there is very little evolution away from the original $\gamma=1$ slope. Evaluated at $\log r=-2$, the slope does show a gradual flattening over the course of the simulation from $\gamma=1$ to $\gamma=0.9$; however, this evolution is consistent with the effects of wandering of the expansion centroid due to root- $N$ fluctuations in the expansion. The low- $N$ model shows stronger evolution, as would be expected if the change in cusp slope was driven by particle noise. This small evolution aside, the model exhibits an unchanging 


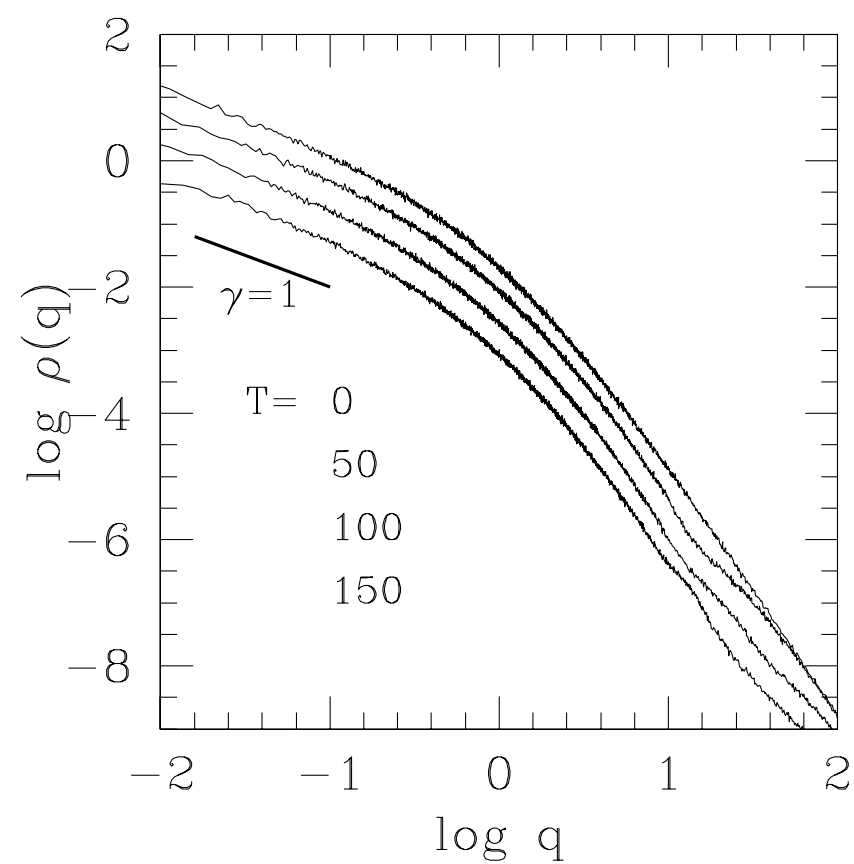

FIG. 1.-Density of the model (plotted as a function of ellipsoidal radius $q$ ) as a function of time during and after the squeezing process. The curves show the density profile at times $T=0,50,100$, and 150 . Curves are offset from one another for clarity. The heavy line shows a $\gamma=1$ slope.

density profile over $\sim 5$ orders of magnitude during and after the squeezing process.

The evolution in figure shape is shown in Figure 3, evaluated at three different ellipsoidal radii. As the first phase of squeezing begins along the $z$-axis, the model becomes very oblate $(c / a \sim 0.6)$. Once squeezing begins along the intermediate axis, the model becomes triaxial, although the short

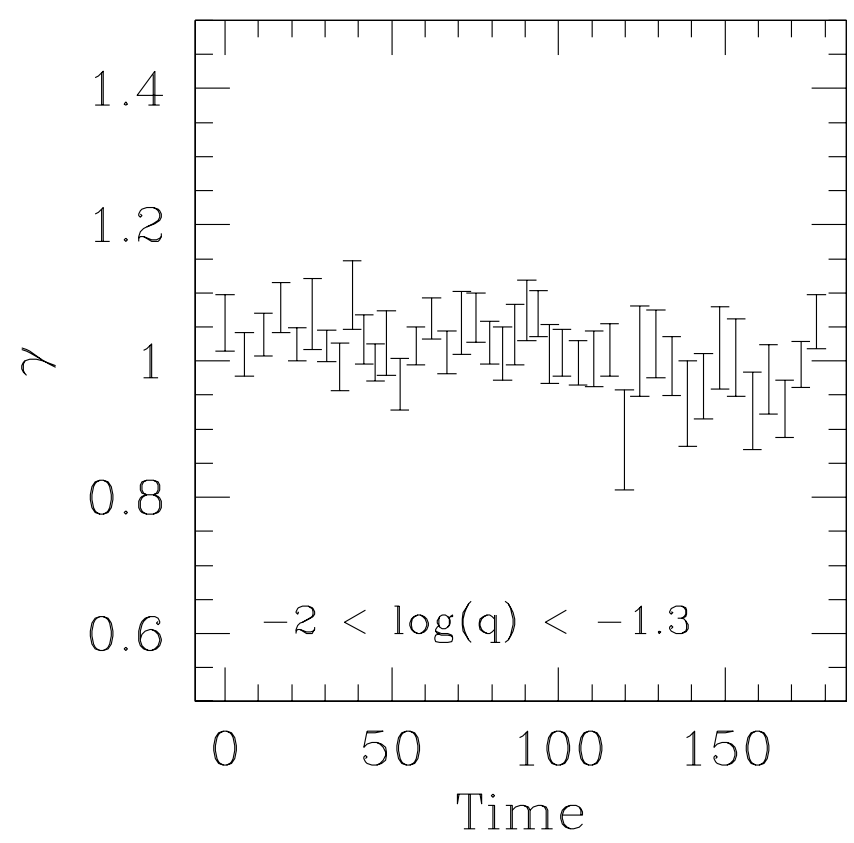

FIG. 2.-Central density slope $\gamma$ of the model, fitted over a range $-2<\log q<-1.3$. The slope is slightly greater than $\gamma=1$ at early times due to the fact that the density profile steepens in the outer portion of the fitted range.

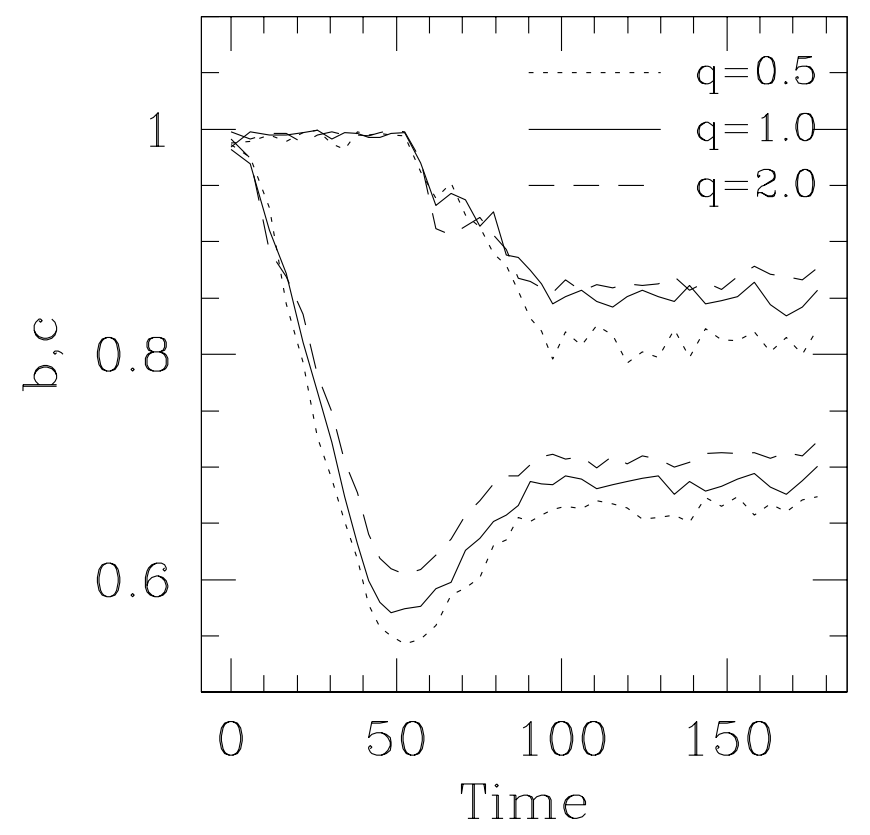

FIG. 3.-Intermediate and minor axes lengths as a function of time. The axes lengths are iteratively calculated from the ellipsoidal density distribution using the moment of inertia tensor. See text for details.

axis rebounds a bit so that when squeezing is complete the final model is only moderately triaxial, with axis ratios $1: 0.85: 0.7$. The rebound of the minor axis may reflect the incompatibility of a $\gamma=1$ cusp slope with a strongly triaxial system (e.g., Merritt 1997; Valluri \& Merritt 1998), as the box orbits that support strong triaxiality become chaotic in a the presence of a strong central cusp. It is not clear that this explanation is correct; given the nonphysical drag and rapidly changing potential, other effects may be in play. To some degree, however, understanding the detailed dynamics at these intermediate stages is rather ill motivated given the nonphysical adiabatic drag we apply. Of more importance is the postdrag equilibrium configuration, which we explore in more depth in the next section.

To explore the variety of shapes that could be constructed using this method, a suite of smaller $(N=128,000)$ models was also calculated in which the dragging coefficients were varied. Table 1 gives the drag coefficients and final shape of these models, while Figure 4 shows the models on the triaxiality plot. The models populate a region of moderate triaxiality, with shapes that range from somewhat oblate to somewhat prolate systems. Ideally, there would be a unique mapping of drag coefficients to final figure shape; this, unfortunately, is not the case. As such it remains difficult to predict the final shape for a given set of drag coefficients. Nonetheless, the calculations do present a set of stable, triaxial, and self-consistent $\gamma=1$ galaxy models useful for studying the evolution of triaxial ellipticals.

\section{INTERNAL STRUCTURE}

Here we look at the internal structure of the highresolution fiducial model in more detail. Figure 5 shows the properties of the model as a function of ellipsoidal radius $q$ $\left(=\left[x^{2}+(y / b)^{2}+(z / c)^{2}\right]^{1 / 2}\right)$ at a time $t=150$, or $7.5 t_{\mathrm{dyn}}$ after squeezing has terminated. For the plots of density and velocity dispersion (Fig. 5, upper left, lower left, lower right), 


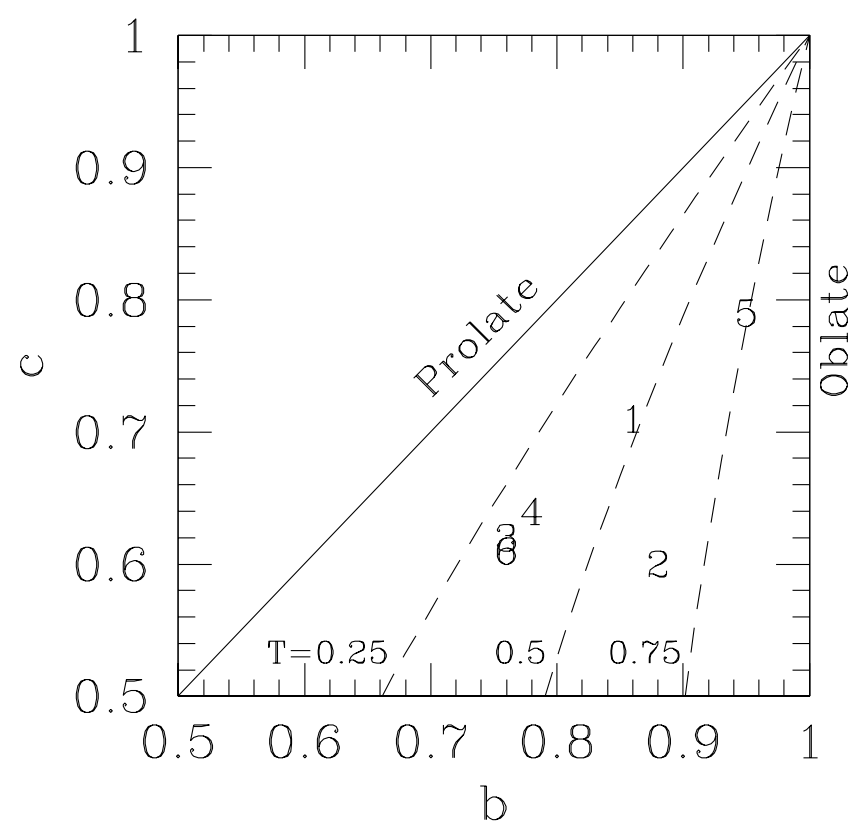

FIG. 4.- Half-mass axes ratios of models generated with different drag coefficients, plotted on the triaxiality plot of Franx et al. (1991).

$q$ is calculated using a constant $(b, c)$ evaluated at the halfmass radius. To calculate shape as a function of radius (Fig. 5, upper right), we use a technique described by Dubinski \& Carlberg (1991), wherein particles are first binned into a spherical shell at a given radius, and the moment of inertia tensor for the binned particle distribution is used to estimate the ellipticity of the particles. The bin shape is then adjusted based on the estimated ellipticity, and the procedure continues iteratively until the bin shape and the ellipticity match.

Figure 5 (upper left) shows that the model retains its $\gamma=1$ character over many orders of magnitude in radius. The model also has fairly constant shape (Fig. 5, upper right), although there is a slight trend for the outer regions of the model to be more axisymmetric than the inner regions. This probably reflects the fact that the squeezing timescale was independent of radius, so that the squeezing lasted for a shorter dynamical timescale at large radius. In the outskirts of the model, the particles have not responded as strongly as those in the inner regions.

The projected velocity dispersion (Fig. 5, lower left) shows a rollover at small radius, as is to be expected from the Hernquist distribution function (Hernquist 1990). The velocity ellipsoid reflects the triaxial shape of the model, with $\sigma_{x}>\sigma_{y}>\sigma_{z}$, where $x$ and $z$ are the major and minor axes of the model, respectively. The model also displays a

TABLE 1

Triaxial Models

\begin{tabular}{ccccc}
\hline \hline Model & $\xi_{0, z}$ & $\xi_{0, y}$ & $b_{1 / 2}$ & $c_{1 / 2}$ \\
\hline $1 \ldots \ldots$ & 0.030 & 0.025 & 0.85 & 0.70 \\
$2 \ldots \ldots$ & 0.030 & 0.045 & 0.87 & 0.59 \\
$3 \ldots \ldots$ & 0.045 & 0.045 & 0.75 & 0.61 \\
$4 \ldots \ldots$ & 0.045 & 0.030 & 0.77 & 0.63 \\
$5 \ldots \ldots$ & 0.010 & 0.020 & 0.94 & 0.78 \\
$6 \ldots \ldots$ & 0.045 & 0.020 & 0.75 & 0.60 \\
\hline
\end{tabular}

slight radial anisotropy in the velocity ellipsoid (Fig. 5, lower right; $\beta=1-\left\langle v_{t}^{2}\right\rangle /\left\langle v_{r}^{2}\right\rangle>0$ ). At large radius $(\log q>1)$, the model becomes strongly anisotropic; for these particles the squeezing has not been adiabatic, as they experienced the squeezing for a fraction of an orbital timescale. In the outer regions of the model, the system has not yet come into full equilibrium, and the model is somewhat suspect. However, the energy and angular momentum content of these particles is large, and they will not greatly affect the equilibrium dynamics in the inner regions of the model. Indeed, the stability of the density profile and shape support this conjecture.

\section{ORBITAL PROPERTIES}

Since the intrinsic shape of any galaxy is dictated by the time average of its orbital content, orbital structure analysis is an essential probe of the viability and stability of any galaxy model. For example, triaxiality in galaxies is supported by the large fraction of stars on box orbits (or boxlets) as determined by the shape and density profile of the potential (e.g., Schwarzschild 1979). The fact that box orbits can travel arbitrarily close to the potential center argues that steep density cusps may destabilize these orbits, seeding chaotic motion and driving galaxies away from triaxiality (Merritt 1997). Indeed, Merritt finds that $\gamma=1$ represents a critical profile and suggests that galaxies with steeper cusps should quickly evolve away from triaxiality. Given that our models represent moderately triaxial systems with $\gamma=1$ cusps, it is interesting to characterize their allowed orbit families and level of chaos. In fact, as we demonstrate below, the equilibrium state of these models displays a mixture of tubes, boxes, and resonant orbits and is not strongly chaotic.

Characterizing the orbital content of these models also allows us to lay the groundwork for future studies of evolution in triaxial ellipticals. The growth of a massive black hole in a triaxial potential can destabilize centrophilic box orbits through stochastic diffusion, driving the global shape of a galaxy toward axisymmetry in a few crossing times (Gerhard \& Binney 1985; Norman et al. 1985; Merritt \& Quinlan 1998; Wachlin \& Ferraz-Mello 1998; Valluri \& Merritt 1998). The change induced in the orbital content (and hence the galaxy structure) by a central black hole will thus be very sensitive to the initial orbit content of the triaxial system. As a case in point, strong shape evolution could be tempered by a population of centrophobic boxlets that could avoid scattering by the black hole, thereby maintaining triaxiality. An analysis of the orbit populations in these triaxial models will help address this possibility and provide an important contrast to the orbital families present under the influence of a central black hole (HolleyBockelmann et al. 2001). For this exercise, it is necessary to determine the initial orbital content and set up the technique here.

Orbit analysis has traditionally been explored in a static, analytic potential. Since an $N$-body representation of the final potential can scatter orbits artificially, it is crucial to reduce the coarse-grained nature of the final model as much as possible. Fortunately, a triaxial figure has a mirror symmetry about each axis, such that a particle at $x, y, z$, $v_{x}, v_{y}, v_{z}$ could also exist at $-x, y, z,-v_{x}, v_{y}, v_{z}$ without distorting the potential (and so on for each axis). We take advantage of this eightfold symmetry by seeding the final state of the model with these symmetric pseudoparticles. 

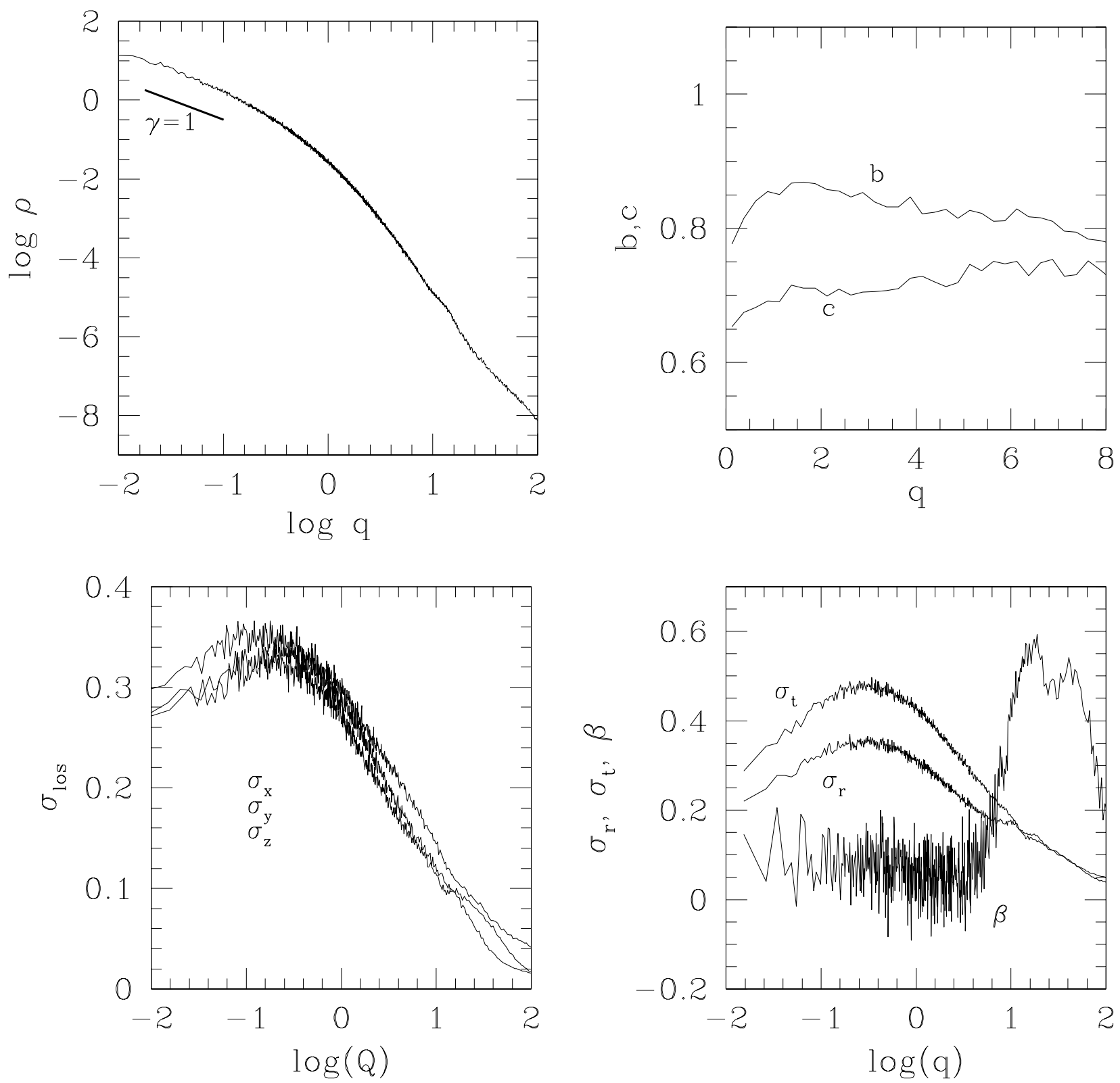

FIG. 5.-Structural and kinematic properties of the model at $T=150$. Upper left: Density profile. Upper right: Intermediate and minor axes lengths as a function of ellipsoidal radius. Lower left: Projected velocity dispersion along the fundamental axes, as a function of projected ellipsoidal radius. Lower right: True radial and tangential velocity dispersion and velocity anisotropy parameter as a function of ellipsoidal radius.

The SCF code was then used with these pseudoparticles to obtain the coefficients for the potential expansion. With the effective particle number increased from 512,000 to over $4 \times 10^{6}$, the noise in the potential was drastically reduced. Hence, all the orbits in this discussion were evolved in the frozen potential dictated by the expansion coefficients from this symmetrically seeded final state.

\subsection{Orbit Classification Techniques}

The large number of particles in each model demands an automated classification technique. In this subsection, we will describe our classification scheme first for twodimensional and then for three-dimensional orbits. In general, we designed a method based on two common classification techniques: axis-crossing pattern recognition and Fourier spectral analysis.
The axis-crossing technique relies on the fact that a regular orbit displays resonances that are recognizable by the pattern that emerges as the particle crosses an axis along its orbit (Fulton \& Barnes 2001). For example, in the simplest case, a tube orbit confined to the $x-y$ plane crosses first the $x$-axis, then the $y$-axis, and will repeat exactly that pattern ad infinitum. Therefore, its axis-crossing pattern will be $x y x y . .$. A boxlet commonly called a "fish" will have the pattern $х у х y х x y x y x . . . ;$ in fact, every planar resonant orbit will have a characteristic axis-crossing pattern. Planar box orbits, on the other hand, are characterized by their lack of a regular axis-crossing pattern. This technique is excellent for the analysis of $N$-body-generated orbits because the act of crossing an axis is insensitive to noise in the potential. Unfortunately, this technique is not useful in discriminating between a pure box orbit and a chaotic orbit since neither orbit has a unique axis-crossing pattern. 
Classification by Fourier spectral analysis is similar in spirit to the axis-crossing technique in that it looks for the characteristic patterns in Fourier space that are generated by a regular orbit. For a regular orbit, the Fourier transform of the time sequence for each degree of freedom results in a spectrum with frequencies that correspond to the quasiperiodic motion of the particle, and the frequencies with the maximum amplitude for each coordinate are the dominant frequencies.

In two dimensions, our technique was based loosely on the Fourier classification scheme of Carpintero \& Aguilar (1998). In short, from the Fourier transform of each coordinate, the orbit is classified by resonances between each pair of coordinates, such that $\left|m f_{1}-n f_{2}\right|>\epsilon$, where $m$ and $n$ are integers, $f_{1}$ and $f_{2}$ are the dominant frequencies, and $\epsilon$ is an accuracy parameter that depends on the frequency resolution and orbital accuracy (due to potential noise and integration errors). For example, the $x-y$ planar tube orbit will exhibit a 1:1 resonance between the dominant frequencies in the $x$ - and $y$-directions, while fish boxlets will have a 3:2 resonance. Planar box orbits will contain many peak frequencies that are nearly as large as the dominant frequency, so box orbits can be identified both by their resonance and by the number of significant peaks.

The classification of three-dimensional orbits is not a trivial "scaling up" of these two-dimensional methods. Since many three-dimensional resonant orbits do not project to an identifiable planar periodic orbit in any plane, the axis-crossing technique may misidentify a resonant orbit (i.e., a 2:1: -2 orbit) as a box (Merritt \& Valluri 1999). This misidentification is important because resonant orbits avoid the center and are thus thought to be more stable against a central density cusp or black hole than a centrophilic box. Fortunately, any stable resonant orbit still obeys a fixed ratio between the dominant frequencies in each coordinate, so on a frequency map (a plot of $f_{x} / f_{z}$ vs. $\left.f_{y} / f_{z}\right)$, resonant orbits stand out as a complex grid-the slope and intercept of any line on this grid identifies the particular resonance (Laskar 1993; Valluri \& Merritt 1998).

Aside from the ability to identify nonplanar threedimensional resonant orbits, the true advantage of the Fourier technique is that it can easily quantify the stability of any orbit. Since the dominant frequency in a chaotic orbit will change with time, it is simple, in principle, to determine the number of chaotic orbits in a given potential. Stochasticity is calculated by the change in the dominant frequency over successive time intervals. So, while the axiscrossing technique cannot differentiate between boxes and chaotic orbits, this Fourier method easily identifies the chaotic orbit as one with a changing dominant frequency. Unfortunately, the Fourier technique is sensitive to noise in a potential, so it is unable to detect a subtle onset of chaos. However, since we are interested in the global stability of our models over many dynamical times, it is sufficient to detect only the stochasticity that is strong enough to change the bulk properties of the model over this time.

\subsection{Methods and Results}

There are two different questions that can be addressed: (1) What orbits are possible in a given potential? and (2) What orbits are actually populated by the particles? The first question can be explored with an analysis of the surface of section, a two-dimensional technique, while the second question must focus on a fully three-dimensional analysis.
We addressed both questions, and this section describes the technique and results of each.

\subsubsection{Two-Dimensional Surfaces of Section}

We integrated orbits with initial conditions designed to evenly trace the phase space along the $x-y$ and $x-z$ planes. There are four sets of $O\left(10^{4}\right)$ particles for each plane, corresponding to binding energies of $E=-1.0,-0.65,-0.40$, and -0.20 and typical mean radii of $\langle q\rangle=0.2,0.6,1.3$, and 3.9. The particles are followed in the frozen final potential and are confined to the initial plane to reduce scattering by noise.

To classify the two-dimensional orbits confined to the $x-y$ and $x-z$ planes, we integrated the particles for up to a total of 50 dynamical times or until $T=8000$, whichever occurred first. ${ }^{4}$ In sets of 12.5 dynamical times (i.e., 50 circular orbits), a particle's trajectory was equally sampled 524,288 times, and the Fourier transform of this time series was calculated by an FFT with a Hann window function. The first two Fourier series were used to determine two independent orbital classifications and each subsequent time series was used only to determine if the orbit had become chaotic. A strongly chaotic orbit was defined in the convention of Valluri \& Merritt (1998) as $\Delta f \equiv \mid f_{1}$ $-f_{2} \mid / f_{0} T>\Delta$ fcrit, where $f_{1}$ and $f_{2}$ are the dominant frequencies at the first and second time intervals, $f_{0}$ is the frequency of a tube about the long axis, and $T$ is the time interval. We set $\Delta f_{\text {crit }}=0.05\left(t_{12.5} / T\right)^{1 / 2}$, where $t_{12.5}$ is the integration time over 12.5 dynamical times. That is not to say that orbits with $0<\Delta f<\Delta f_{\text {crit }}$ are not chaotic; however, the diffusion timescales of these orbits were too slow to produce substantial changes in the shape over 50 dynamical times. Since we are interested only in stochasticity strong enough to induce bulk evolution of the model, we can neglect more subtly chaotic orbits (see also Valluri $\&$ Merritt 1998 for a discussion). The coarse-grained potential also prevented us from distinguishing subtly chaotic orbits from slightly noisy ones, so the detection of mildly chaotic orbits is not reliable.

To complement the Fourier classification, each orbit was also classified according to its axis-crossing pattern. Up to 100 axis crossings were counted to compile the axis-crossing pattern and were compared to template patterns with resonances up to $7: 6$. The higher resonances were relatively insignificant in this model, so they were not tracked. Approximately 200 orbits were cross-checked by visual identification to determine the accuracy of the classification method and to tune the technique, and less than $1 \%$ of the orbits were misidentified in each subsample.

Figure 6 shows the surface of section for each energy slice along the $x-y$ and $x-z$ planes. Notice the profusion of boxes and lower order boxlets in the most bound sets of orbits, characteristic of a cuspy triaxial potential (e.g., MiraldaEscude \& Schwarzschild 1989). In our model, boxlets comprise a larger fraction of phase space along the $x-z$ plane than along the $x-y$ plane. Among boxlets, fish orbits (3:2) are the dominant boxlet on the $x-z$ plane, and pretzels $(4: 3)$ are dominant on the $x-y$ plane. A common boxlet in many analytic potentials, bananas $(2: 1)$, are an insignificant part of this model, most probably due to the relatively modest

\footnotetext{
${ }^{4}$ This hard limit of $T=8000$ was chosen to correspond to approximately a Hubble time of total integration for an elliptical galaxy with $M_{V}=-22$.
} 


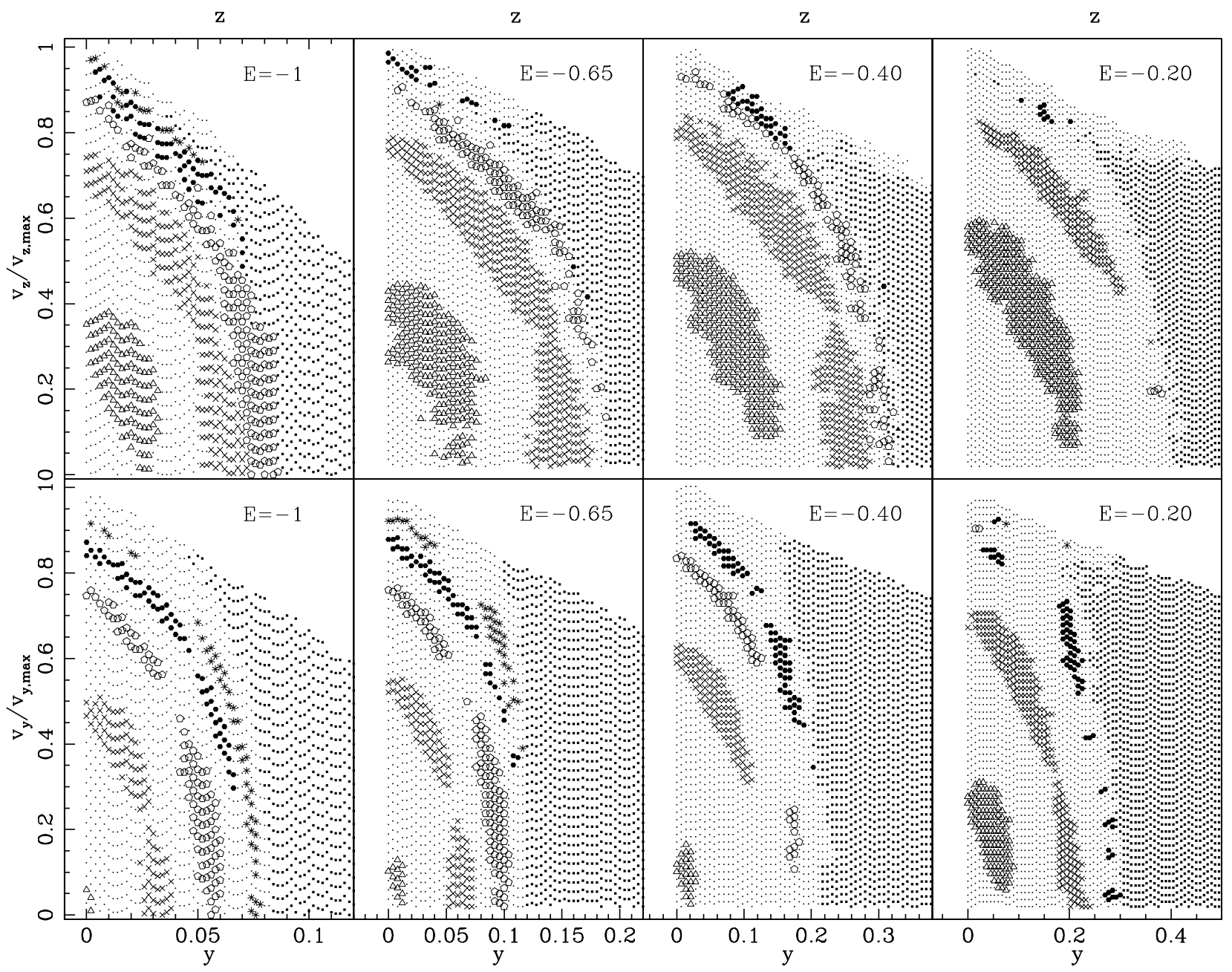

FIG. 6.-Surfaces of section for the triaxial model at $T=150$, plotted for orbital populations of differing binding energies. Top: Surfaces of section for orbits in the $x-z$ plane. Bottom: Surfaces of section for orbits in the $x-y$ plane. Orbits are coded by point type-loops: filled squares; boxes: small points; fish: open triangles; pretzels: crosses; 5:4 resonance: open pentagons; 6:5 resonance: filled hexagons; and 7:6 resonance: asterisks. This plot was created by taking an average of all orbit types at a particular position on the surface of section. We zoom in on the $x$-axis of the plot to show as many box and boxlets as possible; the orbits outside the limits of the plot are all tubes. If plotted to the full extent of the $x$-axis, the boxlet region would comprise $\sim 50 \%$ of the most bound panels and only $\sim 10 \%$ of the least bound panels.

flattening of the figure along each axis (Fridman \& Merritt 1997). On either plane, it is clear that tubes occupy a larger amount of phase space in the outer parts of the potential. In addition, less than $0.2 \%$ of planar orbits were stochastic, as defined above.

\subsubsection{Three-Dimensional Population}

It is also important to know the actual mixture of orbits traced by the particles in the $N$-body model. With this in mind, we initialized the orbits directly from the final state of the $N$-body model. The pool of 512,000 particles were sorted according to binding energy and binned in nine energy slices. As before, particles in the slices corresponding to binding energies $E=-1.0,-0.65,-0.40$, and -0.20 were followed in the final frozen potential, although the orbits were allowed to move in all three dimensions. Since the particles are not confined to a plane, the threedimensional orbits were much more susceptible to noise in the coarse-grained potential, which makes accurate classification more difficult.

Nonetheless, using the same Fourier routine, integration length, and sampling parameters, we were able to extract the fundamental frequencies from these orbits and identify the major resonances using the three-dimensional technique outlined above. Figure 7 presents the frequency map for each energy slice. Notice the strong $(1:-2: 1)$ resonance, which persists from the smallest to the largest radii. In the first two energy slices, many planar boxlets are visible, both in the region around $\left(f_{x} / f_{z}, f_{y} / f_{z}\right)=(0.8,1.0)$ and in the clump around $(0.7,0.9)$. The region at $\approx(0.8,1.0)$ corresponds to orbits that project to tubes in one direction and low-order boxlets, like fish, in another. The clump at $(0.7$, 0.9 ) contains a confluence of planar boxlets, low-order three-dimensional resonances, boxes (which are nonresonant and therefore fill regions off a grid of resonant lines), and orbits that could be identified as either mildly chaotic or noise dominated. In the outer slices, most planar boxlets all but disappear, though the resonant orbit $(2,0,-3)$ is still present. As in the surface of section, the gradual takeover by tubes is apparent in the outer parts of the model, as we go from nearly $60 \%$ tubes (by mass) in the inner region to over $90 \%$ in the outermost slice.

Since a frequency map represents the frequency ratios at a single time, it is not possible to accurately identify stochastic orbits using this plot. Stochasticity for these threedimensional orbits was determined in the same manner as 

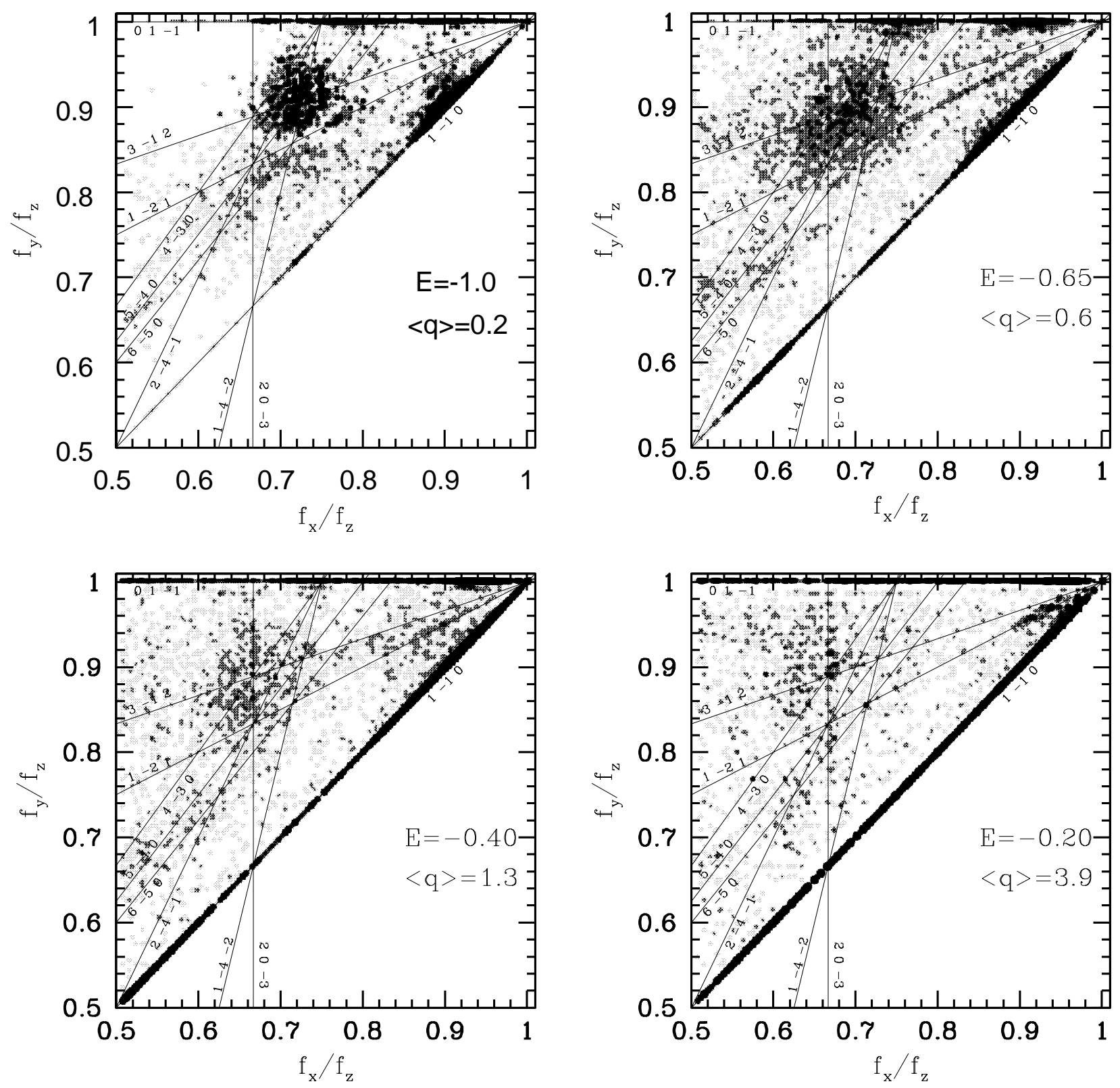

Fig. 7.-Frequency map for the triaxial model at $T=150$, plotted for orbital populations of differing binding energies. The gray scale represents the number of orbits at a given frequency ratio. The lightest gray is one orbit, while black is greater than 50 orbits.

the planar orbits, by determining the change in the dominant frequency over the integration interval. In this stable model, we observe less than $1 \%$ of the particles on substantially stochastic orbits. In fact, the large fraction of tubes versus boxes in our models, a reflection of the modest triaxiality, may preclude the existence of many chaotic orbits. Indeed, nearly every stochastic orbit was found in the innermost slice, where noise from the potential dominates; thus, it is not clear that even these apparently stochastic orbits represent the chaotic orbits found in strongly cuspy, strongly triaxial figures (e.g., Merritt 1997), where boxes are a mainstay. The small number of stochastic orbits found in our model may simply have resulted from noise in the potential.

\section{SUMMARY}

Using a technique we refer to as "adiabatic squeezing," we have constructed models of triaxial galaxies with central density cusps. These models preserve a Hernquist (1990) $\gamma=1$ profile over many orders of magnitude, from $-2<\log (r / a)<2$ or $10 \mathrm{pc}<r<100 \mathrm{kpc}$ when scaled to a luminous elliptical galaxy with half-mass radius $1.5 \mathrm{kpc}$. The models possess fairly constant axis ratios as a function of ellipsoidal radius, showing the range of triaxiality $(0.3<T<0.75)$ and modest flattening inferred for massive elliptical galaxies. The models are stable in isolation, showing little evolution in either the density profile or figure shape over many dynamical times.

A combination of Fourier spectral classification and axiscrossing pattern recognition have been used to classify the orbit content of a characteristic model. At the most tightly bound energies, the models possess a rich variety of orbits, including tubes, boxes, and resonant boxlets, reflecting the triaxial nature and central cusp of the model. Moving to progressively less bound energy slices, the ratio of loops to boxes rises such that the outer regions contain predomi- 
nantly loop orbits. While the presence of noise in the potential expansion limits our ability to detect modest chaos in the orbit populations, we find no strongly chaotic orbits in the model of the type that would result in rapid evolution of the model.

These models thus represent a useful tool for studying the self-consistent dynamical evolution of triaxial galaxies and halos. We are currently using these techniques to explore the evolution of elliptical galaxies harboring central massive black holes (Holley-Bockelmann et al. 2001). Other potential uses include studying the inflow of gas in triaxial systems, the response of triaxial dark halos to baryonic accretion and disk formation, and the infall of satellite companions into elliptical galaxies.

This work is supported through a grant of computing time from the National Partnership for Advanced Computational Infrastructure and the San Diego Supercomputer Center and by NASA through grants NAG5-7019 and HF01074.01-94A. We thank Colin Norman for many useful discussions, and Rebecca Stanek for help with data analysis.
Bak, J., \& Statler, T. 2000, AJ, 120, 110

Barnes, J. 1988, ApJ, 331, 699

- 1992, ApJ, 393, 484

Byun, Y.-I., et al. 1996, AJ, 111, 1889

Carpintero, D., \& Aguilar, L. 1998, MNRAS, 298, 1

Dehnen, W. 1993, MNRAS, 265, 250

de Zeeuw, T. 1985, MNRAS, 216, 273

Dubinski, J., \& Carlberg, R. 1991, ApJ, 378, 496

Faber, S. M., et al. 1997, AJ, 114, 1771

Franx, M., Illingworth, G., \& de Zeeuw, T. 1991, ApJ, 383, 112

Fridman, T., \& Merritt, D. 1997, AJ, 114, 1479

Fulton, E., \& Barnes, J. 2001, MNRAS, in press (astro-ph/0010143)

Gebhardt, K. et al. 1996, AJ, 112, 105

Gerhard, O. E., \& Binney, J. 1985, MNRAS, 216, 467

Hernquist, L. 1990, ApJ, 356, 359 1992, ApJ, 400, 460 . 1993, ApJ, 409, 548

Hernquist, L., \& Ostriker, J. P. 1992, ApJ, 386, 375

Holley-Bockelmann, K., et al. 2001, ApJ, submitted

\section{REFERENCES}

Laskar, J. 1993, Physica D, 67, 257

Lauer, T. R., et al. 1995, AJ, 110, 2622

Merritt, D. 1997, ApJ, 486, 102

Merritt, D., \& Quinlan, G. 1998, ApJ, 498, 625

Merritt, D., \& Valluri, M. 1996, ApJ, 471, 82 1999, AJ, 118, 1177

Miralda-Escude, J., \& Schwarzschild, M. 1989, ApJ, 339, 752

Norman, C. A., May, A., \& van Albada, T. S. 1985, ApJ, 296, 20

Quinlan, G., \& Hernquist, L. 1997, NewA, 2, 533

Ryden, B. S. 1996, ApJ, 461, 146

Schwarzschild, M. 1979, ApJ, 232, 236

Sigurdsson, S., Hernquist, L., \& Quinlan, G. D. 1995, ApJ, 446, 75

Tremaine, S., et al. 1994, AJ, 107, 634

Tremblay, B., \& Merritt, D. 1995, AJ, 110, 1039

Valluri, M., \& Merritt, D. 1998, ApJ, 506, 686

van der Marel, R. P., Sigurdsson, S., \& Hernquist, L. 1997, ApJ, 487, 153

Wachlin, F. C., \& Ferraz-Mello, S. 1998, MNRAS, 298, 22

Weil, M. L., \& Hernquist, L. 1996, ApJ, 460, 101 